\title{
ERRATA, VOLUME 5
}

L. E. Ward, Jr., Partially ordered topological spaces, pp. 144-161.

There are two errors in this paper which were kindly pointed out to me by Mr. I. S. Krule and Professor Russell Remage. Correction is made here and two misprints are noted.

In the proof of Theorem 3, (3) implies (1), each occurrence of the symbol $E$ should be replaced by $E^{\prime}$, where

$$
E^{\prime}(T)=U\{E(t): t \in T\} .
$$

With this change the argument is valid.

Theorem 10, as stated, is incorrect. There is a counterexample due to Remage. It is necessary to add the hypothesis that $E$ be an endelement which is not a cutpoint. The proof, which is believed to be correct, tacitly uses this extra assumption.

By way of misprints, we note the following. On p. 147, last line, read " $E(x) \subset V \subset U$ " for " $E(x) \subset C V \subset U$," and on p. 159, line 7 from the bottom, read " $N$ " for " $n$."

Mark Lotkin, The propagation of error in numerical integrations, pp. 869-887.

The author wishes to insert a note that this paper is a result of the research which he did while he was associated with the Ballistic Research Laboratories.

\section{ERRATA, VOLUME 6}

O. Litoff, On the commutator subgroup of the general linear group, pp. 465-470.

p. 465 , line 16. The parenthetical expression "(except for the case of noncommutative division rings)" should be omitted.

p. 466. Footnote 1 should read "Dieudonne in [4] extended the definition of determinant to the case in which $R$ is a noncommutative division ring. All statements about $U$ in this paper remain valid if this definition is used. (In particular, Theorem 9 can be proved by showing that $D(\mu) \in \mathcal{T}$ if $\mu$ is a commutator in $R$.)"

Abe Shenitzer, Decomposition of a group with a single defining relation into a free product, pp. 273-279.

p. 274. After line 4 insert "Mappings of the form $a_{i} \rightarrow a_{k}^{ \pm 1}$ are also classified as (absolute) level transformations."

p. 276 , line 20 from the bottom. For "a level" read "a (nonabsolute) level."

p. 276, lines 15,16 , and 17 from the bottom. The parenthetical expression "(if $V$ is trivial, i.e. $L(V)=1$, this statement is obvious; if $V$ is nontrivial the statement follows from our lemma)" should be omitted. 